\title{
Resolving Incommensurable Debates: A Preliminary Identification of Persona Kinds, Attributes, and Charac- teristics
}

Ingbert R. Floyd', M. Cameron Jones², \& Michael B. Twidale', ${ }^{1}$ Graduate School of Library and Information Science, University of Illinois at Urbana-Champaign, Champaign, IL, USA \& ${ }^{2}$ Yahoo! Research, Santa Clara, CA, USA

\begin{abstract}
Persona-based design (PBD) has become a popular method for enabling design teams to reason and communicate about user-centered design issues and trade-offs. There is a growing body of literature that describes different ways in which personas have been applied by researchers and practitioners. Despite this diversity in practice the debates about the usefulness of PBD as a method treat it as a single design method that is either good or bad. As a result, the present authors feel it is important to look more critically at what different authors are doing when claiming to use the persona concept, and to develop a theoretical distinction between various persona kinds and their attributes, as well as different characteristics which individual personas may exhibit. This method of analyzing the creation of personas, they believe, can be applied to other design techniques, in order to gain a better understanding of how they work, and how different methods of application can have different consequences for the resultant designs.
\end{abstract}

Keywords: evolving methods, personas, persona-based design

\section{INTRODUCTION}

It is not uncommon in research for different disciplines to use similar or identical terms to represent very different concepts. Once these translation problems are discovered (often via miscommunication) it typically is easy to identify and clarify them in academic writing because different senses of the same term draw upon different literatures and references, thus making the distinction apparent. However, a more subtle kind of miscommunication frequently goes unnoticed. Often, different people will appear to be talking about the same thing, using the same labels, referencing the same papers, and making claims which seem to build upon each other's work. Yet, there exists a misalignment: the supposedly unitary thing being discussed is actually a red herring; in fact, more than one thing is being referenced. Identifying and resolving these differences can be challenging but very important. The differences often make the uses of the particular term incommensurable; the ideas have evolved in such different trajectories that the only similarities they retain are in name and origin. We have seen this kind of divergence in the use of personas as a design tool. To address this divergence, we have performed a careful analysis of the multiple ways in which persona- based design (PBD) has been conducted in the research literature, from which we have formulated a common language for talking about different kinds of personas and the attributes of those kinds, as well as for interpreting the design and research which purports to use them via an analysis of the characteristics particular instances of personas can have.

In their call for papers, the editors of this special issue made a distinction between research-into-design, research-for-design, and research-by-design (Lowgren, $\&$ Nagai, 2008). This paper addresses the researchinto-design portion of the typology. However, instead of examining how to use personas effectively, how to improve the utility of PBD, or how personas are actually used by designers, this paper takes a more abstract perspective by examining researcher practices and vocabulary by studying the research-into-design literature (rather like meta-reviews). The product of this work is not a "finding" about personas (the object of study) or PBD; rather it is a finding about the theory and practice of the people who study personas. Thus, we are engaging in building the intellectual infrastructure to support research-into-design.

\section{THE PROBLEM WITH PERSONAS}

Personas are "hypothetical archetypes of actual users" (Cooper, 1999, p. 124) used throughout the design process to represent those users and their anticipated reactions to design elements in circumstances of actual use. A persona description typically consists of a name and a description of interests, goals, life circumstances, appearance, interests, and preferences. The description may also include a photograph, or at times a sketch. The major function of a persona is to enable designers to break free of their natural tendency to design for themselves.

It is difficult to design for another person. One must develop an empathetic internalization of his/her 
understanding of the world, his/ her experiences in it, etc. It is even harder to design for a collection of other people with all their subtle variations. Thus, designers revert to designing for themselves without really thinking about it, which is facilitated by humans' natural tendency to assume that other humans think exactly like they do, until there is evidence to the contrary. At best, designers may design for their personal stereotypical conceptualization of the "user". Personas help avoid this tendency because they make a group of users concrete in the form of a fictional, single user for whom the designers are designing; thus they are a handy tool to facilitate user-centered design.

PBD was popularized by Cooper (1999). Cooper (2003) claims to have originated the persona concept and is widely credited as the originator and the one who elaborated and popularized personas through his 1999 book. However, some elements of the persona concept had been used by other practitioners and researchers. For example Lafraniere (1996) notes the use of named characters in describing use scenarios: "This method seems to help users more clearly state their work and their relationships with other people." Lafraniere's "proto-personas" are not fully fleshed out, and are mostly defined by role and activity rather than by interests or goals, but even this minimalist use was found to have certain benefits. What Cooper indisputably created was a sophisticated and specific method of generating and using personas in design work (Cooper, 1999, 2003; Cronin, 2005). This fact seems to have been lost by many of his imitators, and by many critics of the method.

The murky origins of personas and the history of related developments that predate Cooper both contribute to the multiple interpretations of personas which exist today. Methods that might be criticized as poor imitations of Cooper's method may actually have legitimacy as extensions of earlier forms, although this is an aspect we do not address in detail. In the following discussion, we will identify and name some of the different interpretations of personas. We acknowledge Cooper as the source of a true PBD method, and evaluate most other methods of developing and using personas as variations on, or derivations from, Cooper.

Personas are used to create scenarios of use (Cooper, 1999, pp. 179-200). Scenarios have been used in design for a considerable time, predating the articulation and elaboration of the concept of personas, and significant research has been done in scenario-based design (SBD) (Carroll, 2000; Rosson \& Carroll, 2002). SBD is similar to $\mathrm{PBD}$, and the two are not mutually exclusive. The two mostly differ on their focus and on the speed of generation of scenarios. For PBD, not surprisingly, the generation of the personas is the primary activity. Once generated and shared with team members, the personas can be used to develop a range of scenarios. The existence of clearly understood personas makes it possible to generate numerous use scenarios very quickly, and then to judge easily which of these are more or less plausible activities for various different personas. By contrast, SBD typically starts with an envisaged scenario that is then fleshed out, analyzed, and critiqued. Many scenarios developed without explicit use of PBD still include elements of persona use as seen in the details of the description of the users, their motivations and actions as embedded in the scenario description. The use of such details can lead to similar advantages (as outlined below) to methods that more explicitly use PBD.

PBD is useful for identifying key attributes of users and capturing them in such a way that they not only facilitate communication between designers, clients, and potential users, but also facilitate decision-making and evaluation by avoiding the trap of designing for the generic "user", or for the developer to end up explicitly or implicitly designing for him/herself. In Cooper's (1999) introduction of the method, he provides specific implementation requirements for creating and utilizing personas. Since then, various researchers (e.g. Grudin \& Pruitt, 2002; Sinha, 2003; Aquino \& Filgueiras, 2005; Pruitt \& Adlin, 2006; Jones et al., 2008) have explored using personas in many different ways. The manner in which these researchers have chosen to implement personas has sometimes radically diverged from the method specified by Cooper (1999) and other practitioners of the original method (e.g. Cronin, 2005) in their publications.

The HumanComputer Interaction ( $\mathrm{HCl}$ ) literature tends to treat the use of personas as a single design method. While some researchers acknowledge that different groups make different implementation decisions (e.g. Rönkkö et al., 2004; Norman, 2006), the discussions they present still seem to presuppose that the use of personas represents a single method, which is tweaked a bit each time it is used. It is not controversial that any application of a design method will be customized to the needs and circumstances of the design team utilizing the method. However, we believe that in the case of personas, the changes made when applying the persona concept go beyond these local variations and involve methodological changes that not only significantly affect the general utility of the concept, but also have implications both for the kinds of situations and the kinds of uses for which the particular implementations are appropriate. Thus, we believe that several different methods that are appropriate for different contexts are all being grouped together under the PBD name.

Recently, stark critiques of PBD as a method have been published (e.g. Chapman \& Milham, 2006; Portigal, 2008), as well as critiques of how the method is often applied (e.g. Marshall, 2003). These critiques are at times intended, and are frequently interpreted, as being aimed at the whole idea of using personas in design. In part, this paper aims to counter the argument that "personas are bad" by showing that it all depends on which kind of persona you are using, how you are using it, and what you are using it for. That is, although there is merit to 
some of these criticisms, others are misguided because they fail to take into account the diversity of activity that is labeled PBD. In this paper, we have decided not to decompose each criticism and identifywhich is correct andwhich is misguided, an activity we believe any individual who is familiar with the persona literature can conduct with ease. Rather, we believe it will be more productive to defend the method indirectly by identifying the true diversity of how personas are utilized in practice, and explore the affordances of each kind of use.

Our motivation for this paper is that we see the concept of PBD potentially following the "Life cycle of a silver bullet" (Sheard, 2003), and we wish to halt this downward cycle. In that paper, Sheard shows via an engaging story how a management method or a software development method can go through a series of phases in adoption and use that progressively degrade its utility and reputation until it is discarded as yet another fad. In brief, a method is developed in-house, refined, publicized, replicated, and evaluated, showing significant benefits. The method is then proceduralized by others who need to cut the costs of applying the method, but without access to the original developers and their detailed experience of creating, refining, and using the method. In doing so, much of the underlying spirit and power of the method is lost, although the name persists. Sheard's warning fable can be used to describe the unfortunate translation of personas from a robust, although subtle and complex, design tool into a routine, checklist-type implementation and on to a self-serving justification that is unlikely to deliver the powerful results of the original versions.

Additionally, in the context of PBD, many implementations we see in the literature (and many implementations that critiques are aimed at) are these later, proceduralized versions of the method. This is a shame given the power and flexibility of personas as a construct and PBD as a method, and we fear that it is unfair to fault personas generally with the problems stemming from local applications of specific instances of the concept. We are not claiming that there is one right way to do personas whereby deviations from this method are degradations that are inevitably problematic, bringing the full method into disrepute. Although this may happen, there are also perfectly legitimate developments and variations that have been designed to meet the constraints of a particular context and in so doing may explicitly trade off certain benefits of the original method for lower cost or better focus on a subset of uses. As you read this paper, many of the examples we present will probably cause you to think that the designers who have created them were not using personas correctly, or just "don't get it". This may be true; however, this distinction is often overlooked in critiques of the method, which take poor persona implementation as reflective of the concept as a whole.
It is both a strength and a weakness of PBD that the idea seems so appealing that many people choose to adopt it and knowingly or inadvertently modify it in both positive and negative directions. This power of the method does expose a danger that various critics note - that it is dangerous in the wrong hands, using the aura of the technique to justify slapdash, lazy, and selfserving design ignoring the study of real users (Portigal, 2008), and so excluding the use of other usercentered approaches - ironically those which Cooper advocates $\mathrm{PBD}$ as a means to support.

In order to make sense of the field of persona use, and to establish a framework for evaluating the effectiveness of particular implementations of personas, we present a preliminary identification of persona kinds, attributes, and characteristics. It is our hope to dispel some of the reservations people hold against personas by shifting the attribution of the negative outcomes and criticisms of personas away from the general concept and onto specific types of personas and the implementation decisions of the designers and researchers who use them. Ultimately, this paper is an attempt to reshape the debate on personas, by focusing the discussion on the particularities of individual implementations so that we can better understand where utilizing the concept is appropriate and productive, and where it is less appropriate and less productive.

\section{THE PERSONA CONCEPT: \\ WHY AND HOW IT WORKS}

Grudin and Pruitt (Pruitt \& Grudin, 2003; Grudin, 2006) make a strong case for the reason why personas are so effective (when used properly), a case which makes concrete the assumptions and implications present in Cooper's (1999) presentation of the method. We elaborate on it here, because we think that accounting for the mechanism is critical to address much of the skepticism encountered, and indeed serves to help account at least in part for why there is so much skepticism in the first place. Their argument is based on the psychological theory of mind, which states that humans inherently and automatically model the mental states of other humans they are around, and use it to predict their behavior. Referencing this theory, they claim: "Personas invoke this powerful human capability and bring it to the design process. Well-crafted Personas are generative: Once fully engaged with them, you can almost effortlessly project them into new situations" (Pruitt \& Grudin, 2003, p. 12).

The generative power of personas is what makes them useful in the process of doing design, where very many decisions have to be made at all stages and all levels of analysis. It is not possible to read the relevant literature on, rigorously analyze, experiment, or even discuss each decision. What is needed is a quick way of guiding the evaluation of costbenefit tradeoffs of alternative points in an immense design space. A brief consideration of questions like: "Which one will John and Mary like 
best? Will Alex cope with that? Is that something Jane will use?" can help guide decisions that otherwise will be somewhat arbitrary, and can turn out later to be problematic. This may not be the most rigorous or definitive of methods, but it is fast enough to compete with the most likely alternative: that of developers making decisions based on their own assumptions, typically how they would use the system.

This works because a persona gives us an operational mental model of a particular kind of user. As such, it can be thought of as a folk version of the models psychologists construct of human reasoning that can be used to predict certain behaviors. In HCI the GOMS model is perhaps the best known. Such a model can effectively predict problems that people are likely to have with using a particular application's interface. For example, most psychological models would predict problems in the use of an application that requires users to simultaneously have nine separate things in their working memory. In a similar way, thinking about a persona using an application can help a designer predict some (but not necessarily all) likely problems - subject to various caveats we explore below.

In many ways personas can be more powerful than psychological models, however. Human beings are intrinsically social animals. As such, we have welldeveloped abilities to reason about other humans, and the psychological literature has demonstrated that we have and develop these skills at avery early age.We use this theory of mind very successfully in our everyday lives when trying to predict what a particular person might do in a certain set of circumstances. Personas draw upon our ability to reason easily about other human beings, thus they harness the full power of our evolutionary heritage that developed to understand our fellow humans (Grudin, 2006). Psychological models often focus on specific features of human cognition, while overlooking other features. Thus, personas are more general in their coverage, and can allow us to reach understandings which strict adherence to existing psychological models may be unable to provide. Furthermore, they can allow us to reach such understandings with considerably less effort, because most of the information processing happens subconsciously. Psychological and sociological models are still useful, because they are particularly suited for catching aspects of human cognition and activity which our natural intuitions do not capture. However, we suggest that good design needs to draw upon both our evolutionary and our scientific heritage.

Using personas provides designers with an alternative to the typical practice of referring to all people who might use a particular product or service as "users". While there are other ways of talking about "kinds" of users, the advantage of using personas is that they evoke the social intuitions and empathies of designers in ways that conversations about nameless, faceless users do not (Cooper, 1999; Norman, 2006). When we start thinking in abstract terms we do not draw on our social intuitions as strongly as we do when we are talking about particular people, especially people we know. Thus, personas work by encapsulating information about potential users of the product or service being designed into a single character who has a name, a face, a history, and, most importantly, a personality. The result is a construct which draws out our social and emotional intuitions much like a fictional character in a novel or play.

Pruitt \& Grudin (2003) note the great power of fiction to engage us, and hence to communicate. The comparison to literature is not accidental: effective personas must be believable characters, and thus multidimensional. The two-dimensional character who is much maligned in high school English composition classes cannot serve as an effective persona (at least, not in ways in which personas are typically used) because, while his/ her actions can be predicted, these predictions are based on social conventions and social scripts, not social intuitions (see Table 1 for an illustration of the difference). This may also account for why additional information about the persona (appearance and elements of a back story) seems to be so powerful, even if it does not appear to have any immediate relevance to the process of design. For example, some aspects of the persona's life, interests, and preferences can be seen to be at least possibly relevant (has a computer at home, is a working mother, uses online health information, hates computer games) while other parts are not (has short blonde hair, likes dogs but not cats, grows carnivorous plants), but can help in rounding out the persona, making her more vivid and hence more memorable.

In literature, once an author has created a rich believable character, it is possible to place that character in a new situation and imagine how she would react. This can often be in ways that are inconvenient for the overall envisaged plot, but an author who coerces a character to act out of character is at great risk of destroying the believability of his fictionalworld and losing the trust of his audience. Authors frequently remark on how their fictional characters seem to take on a life of their own (Grudin, 2006). The importance of staying true to the character also applies to persona use. Once personas are developed, the power of the method is seriously degraded and often destroyed if the personas are coerced to act out of character to fit the overall "plot" of the design team. However, if done correctly, "the power of storytelling may be the single most important reason why personas work" (Quesenbery, 2006, p. 554).

Persona use appears to work through particularity thinking about a particular person, even if fictional. By contrast there is substantial evidence thatwe have great difficulty thinking in terms of generalities and abstractions. Processes such as Boolean logic, computer programming, and indeed academic research, for which abstract thinking is essential, are difficult. Ability in them is only acquired through much practice and effort - and so it is not surprising 
that people who have achieved such skills are proud of their achievements and perhaps a bit suspicious that a method based on the premise of the antithesis of abstraction can possibly help them with such abstraction-essential activities.

Our social intuitions consist of many parts; some are hard-wired, some are developed from the experiences we have in our life, some are developed from our conversations with others about howother people think and act, and most are some combination of all three. Via our social interactions, we encapsulate some of the social intuitions we have about the regularity of certain groups of people's behaviors into culturally shared generalizations which can be more or less founded in actual experience. Such generalizations are what we often call stereotypes, and experientially derived stereotypes can be useful in persona development. It is important to be cognizant of the stereotypeswhich are selected, and the implications of selecting them, in order to avoid ethnic, gender, or status profiling. Properly used, however, they can still serve as a powerful mental "subroutine" encapsulating a significant amount of shared intuitive understanding of the world between designers. This is one of the most important mechanisms bywhich personas operate. Instead of talking about "users", a term which allows the designer to project his or her own (or any other) personality onto the target population, they talk about personas which, if written well, generate social intuitions which the designer cannot ignore, thus allowing the designer to predict the behavior of a user even if such behavior is not how the designer him- or herself would act.

Of course, many engineers reject personas for subjective reasons. Personas sound vague, silly, or bogus. They just seem to be a way to let English majors play at creative writing. They aren't about nice, safe, hard things like data and numbers. Therefore, they treat personas with extreme skepticism. For this reason, it is possible to fail to integrate personas fully into a design process, and so fail to get the advantages claimed for them, particularly if the designers do not trust the persona (Blomquist \& Arvola, 2002). To address this, Cooper (1999) emphasizes the importance of ensuring that the whole design team understands the nature and value of PBD to obtain the buy-in necessary to get PBD to work. When such efforts fail, then there is a high risk of PBD failing as a method (for examples, see: Rönkkö et al., 2004; Rönkkö 2005; Williams, 2006).

In use, personas frequently act as a useful negative force, encouraging or allowing designers to consider what not to implement at least as much as what to implement. One component of this is to combat the temptation to accumulate an ever growing list of requirements that large numbers of potential users might possibly want. This applies to all product design but is particularly acute in software design where the addition of yet more functionality can seem particularly tempting as away to improve the appeal of the product, whereas in
The classic 2-D example:

The evil villain tries to kidnap the helpless girl so that he goes around doing evil things like clear-cutting a forest for personal gain while the hero is distracted trying to save the girl

The multidimensional version of the "evil" villain has more complicated, believable notions that call into question his "evil" status:

A man who has built a logging company from scratch, starting with a two-person crew, and growing it into a company that is the major employer in his region, is facing off against an environmentalist who is concerned about the destruction of oldgrowth forests. In desperation, after the environmentalist has sabotaged his machinery multiple times, and he is worried about bankruptcy, and putting all of his employees out of work, he decides to kidnap the environmentalist's fiancée, to distract the environmentalist away from sabotaging the last four treecutting machines he has left

Table 1.

Character dimensions

actuality it bloats the application delaying the delivery of a robust reliable product and bewildering users with too many options. Personas allowdecisions to be made about which features and functionalities to drop, and which need to be optimized to meet actual needs. This focus on the negative (what the personas do not want to do, what they dislike, what they struggle with in software) is very valuable in informing design decisions and redesign prioritizations, but is at odds with the necessarily more positive, optimistic needs of other related activities such as market segmentation characters used in marketing.

\section{THE MOTIVATION}

The above account describes the reason behind the power of personas, at least with regard to how they were originally developed and used by Cooper and others. As suggested at the beginning of this paper, however, we have found that particular accounts of personas in the literature seem not only to contradict each other, but to be talking about totally different things, despite using the same word ("personas") to identify these things. To demonstrate our point, we will briefly review some selected accounts of personas we have encountered in the literature. However, rather than focus on each account individually and then provide a comparison, we will instead focus on one of the major variables by which personas differ: the source of the information in the persona.

The source of the information in the persona tends to be the variable that causes the most controversy. In Cooper's original conception, he clearly states that personas must be formed based on ethnographic research of users (Cooper, 1999; Cronin, 2005). The purpose of the personas that designers develop with Cooper's method is to capture the intuitive understanding of the user in his/her environment that the designers gain via ethnographic study, which is hard 
to articulate via other means. Cooper's personas are thus primarily based on empirical evidence. Any fictional components of the personas are there only to spark proper intuitions about the users being designed for. Many people who have built on Cooper's work have kept this strong empirical bent, even if the sources of their empirical data are different from Cooper's ethnography (e.g. many different types of empirical data were used by Pruitt \& Grudin, 2003 and Pruitt \& Adlin, 2006; surveys were used by Sinha, 2003). They tend to feel that the strict empiricism is what keeps the method rigorous.

Not all personas are strictly empirical, however. Norman (2006) describes the ad hoc persona, which is a fictitious persona developed by designers to capture their intuitions about certain aspects of particular users' behavior (often during conversation). These have their own value, not in capturing actual user needs, but in capturing the designer's intuitive understandings and making them available for discussion in ways in which pure description would not. Djajadiningrat et al. (2000) also create fictitious, ad hoc personas in the form of their extreme characters, personas created to explore the edges of the design space, and to raise issues which otherwise would not be addressed by simply considering the "standard", representative personas of Cooper, Pruitt, and others. None of these fictitious personas claims to be based on empirical reality, except as they reflect the designer's own intuitive understanding of such reality. In fact, while the ad hoc personas Norman describes are intended to be believable and reflect reality, Djajadiningrat et al. make no effort to keep their personas real or believable; instead, they are created to push boundaries and expectations to see what kinds of activity a design can handle, or to expose other ways in which a design could be optimized.

The empirical source of persona data tends to be the flashpoint for most of the major criticisms of personas as a method. Almost all such criticisms cite a lack of or an insufficient empirical grounding as a reason why PBD fails. The best, and most balanced, critique is by Marshall (2003). Marshall clearly addresses personas as actually used by designers in certain organizational settings, not personas as depicted in the research literature (primarily those developed and used by their inventors). She not only demonstrates the kind of information which personas often do not have, but also how easy it is to run with fictional personas, to morph them into exactly the kind of elastic user that Cooper proposes personas as a remedy to avoid (Cooper 1999). However, she suggests that the antidote to this is not to give up on personas, but rather to integrate ethnographic observation of various sorts into one's work. In particular, she suggests a life-as-informal-ethnography she terms "feral ethnography" as a quick and easy way both to build awareness of what people actually do, and as a way of building intuitions which will not only allow more robust applications of personas, but also criticisms of those applications which are misguided. Portigal (2008) seems to be motivated by similar observations of actual persona use by designers, but his reaction is much stronger. He sees designers as fabricating personas because it is easier than doing research on real users. His solution is to ban the use of personas, which is neither feasible nor likely to happen, and disregards the real benefit they can provide to a design project.

Both Marshall's (2003) and Portigal's (2008) criticisms raise valid concerns. The personas they are encountering are not ad hoc personas, because the function they are being called upon to perform is to represent real users in all of their complexity, not to serve as communicative means for expressing the designer's intuitions, or to test the limits of a design space. Thus, these personas are being created to serve a role that is more appropriately filled by the kind of personas created by Cooper or Pruitt. Stretching the persona beyond what is warranted by empirical evidence, not collecting diverse enough kinds of empirical evidence to fully flesh out the persona, and failing to collect any empirical evidence at all are all inexcusable lapses in the application of PBD as a method. However, these are clearly the kind of methodological corruptions that Sheard (2003) warns about, and do not constitute problems with PBD as a method itself, as Marshall recognizes, but Portigal blithely ignores.

The other major criticism of PBD is by Chapman and Milham (2006). Their criticisms are purported to apply to PBD in general; however, the only PBD method they address is that set forth by Pruitt and his collaborators (Grudin \& Pruitt, 2002; Pruitt \& Grudin, 2003; Pruitt \& Adlin, 2006). This is telling, because most of the criticisms they put forth do not apply to PBD as set forth by Cooper (1999), Cronin (2005), Norman (2006), etc. It is beyond the scope of this paper to address all of their concerns with respect to the major methods of PBD present in the literature; however, we will address some salient issues here. Personas are not intended to be direct representations of the user. The whole purpose of Cooper's method of aggregating initial candidate personas together into a smaller set of more accurate personas is to create an abstracted representation of the user. Thus, it is a fundamental misunderstanding of the construct to claim that the individual features of a particular persona need to be present in the users it represents. Personas are not statistical abstractions and instead of being a measure of central tendency, personas are a representation of an abstract class of users. The individual characteristics and traits of a persona spark a similar kind of intuitive understanding of a user's perspective and experiences that one would get from performing naturalistic observations of real users. Thus, personas are a design technique, not a technique for scientifically understanding or representing the world, and do not need to be falsifiable; they just need to work. Furthermore, they are a tool, one tool among many. How does one evaluate whether a flathead screwdriver is better than a Phillips head screwdriver? One doesn't. Rather, one picks the one that is available and suited for 
the job at hand. As long as the job gets completed, which screwdriver is selected is moot. And there is evidence to show that personas do contribute to successful design, i.e. they work.

This is not to say that there are no valid criticisms of published PBD methods. For example, Sinha (2003) has developed a frequently cited method of generating personas via quantitative analysis of survey results. However, while the technique is interesting, we have serious concerns about whether the survey results he generated actually represent any meaningful qualities of possible user populations. While we could see his method as a possible supplement to the Pruitt approach to PBD, we are skeptical of its standalone value, as we do not see how most surveys could reveal the same kind of insights into user experience as methods such as ethnography can. Of course, Sinha's work has not yet been vetted in real-world design projects as other methods of PBD have been, so perhaps more research is required.

\section{A PRELIMINARY IDENTIFICATION OF KINDS, ATTRIBUTES AND CHARACTERISTICS}

We have created a preliminary description of persona kinds, attributes, and characteristics based on the many different ways in which people (researchers, designers, marketers, students, users, etc.) construct personas. This ontology' is intended to highlight which information is utilized in order to create personas, and the implications of constructing personas in this manner. We drew upon several sources when compiling this ontology, including: published personas and reports of persona usage; our personal experiences creating, using, and observing the creation and use of personas; and our experiences teaching personas and using them in the classroom (see Jones et al., 2008). By outlining this basic ontology of personas we hope to accomplish several goals:

- to point out several common pitfalls or problems in the application of personas;

- to promote conversational clarity about the different nature of personas as actualized from the general persona concept;

- most importantly, to initiate a more formal discussion of personas as a tool in design.

The ontology we set forth is meant to be a reflection of real-world uses of personas. As such, like a phylogenetic taxonomy, it is as complicated as the range of designers' activity requires. It is not a tool to reduce the cognitive burden of the designer trying to understand personas. As desirable as such a tool would be, we could not find patterns either in the literature or in our prior experience which lent themselves to such an account. Rather, our ontology does the opposite: it is meant to be a reflection of the world as-is, not a simplification that expedites human cognition.
New forms of conducting PBD will probably require new persona kinds to be added to the ontology. However, this is a positive feature, as the ontology is able to reflect the fact that the new persona kind is constructed under assumptions and with structural consequences that are unlike existing personas. Thus, when new personas are encountered in real-world design activity, personas which do not cleanly fit within the ontology as it currently exists should raise a red flag. This means that they should not be treated as unproblematic implementations of a well-understood method. Rather, the logic behind their construction, the consequences of their formation and structure, and the benefit they are intended to provide should be carefully considered so that the value and validity of the new method is checked and verified. Presence in the taxonomy simply implies a precedent which can be consulted where at least some of this work has been done. And if different personas fit into different kinds, then it suggests that lauding or criticism of one kind of persona does not necessarily apply to another kind of persona. Any criticisms that are intended to apply to all personas need to be examined in light of the underlying assumptions and features of each of the personas in turn.

The identification of persona kinds, attributes, and characteristics was motivated by the nature of the data (the persona literature, our experiences using them, etc.). At first, we were simply trying to create a typology of personas to better distinguish the different kinds (or types) of personas we were identifying to bolster our observations of miscommunications in the literature. However, as we proceeded with the analysis, we found that some kinds of personas overlapped, other kinds seemed mutually exclusive, and that possible ways of grouping persona kinds were not as cut-and-dried as it first appeared. For example, as can be seen from the discussion above, "empirical" personas and "fictional" personas are not as clearly distinct as providing them with different types in a typology would suggest. Even empirically grounded personas often have fictional components to them. The real question is: What aspects of the particular persona are fictional, what are empirically based, and what follows from this mix of fact and fiction? For this reason, we recognized that more subtle distinctions needed to be made.

Currently, there are three ways in which we describe personas. First, there are what we call the persona kinds. These are tightly tied to the particular PBD method - change the method, and you change how the persona is constructed, its implications for design, etc. Then there are persona attributes, derived generally from the major ways in which persona kinds differ from each other. Persona attributes modify persona kinds: each kind of persona has its attributes which describe it. Finally, there are descriptions of personas which do not classify personas, but rather identify individual personas as having certain features, structure, etc. These we call persona characteristics, to distinguish them from 
persona kinds, which identify aggregates of one sort or another.

\section{PERSONA KINDS AND METHODS OF PBD}

Persona kinds are inextricably linked to PBD methods. Every PBD method has certain intents which motivate the creation of a persona, certain sources of empirical data, constraints about what components of the persona can be fictional, constraints on how a persona can be properly used given how it is constructed and structured, etc. Each of these components is handled by different persona attributes (discussed below). Together, however, they specify a persona kind, which functions in particular ways. The purpose of distinguishing the kinds is to highlight the fact that the personas created by different PBD methods are to be used in different ways, and thus they cannot always be directly compared with one another meaningfully. Any implementation of PBD must specify which persona kinds it is creating, and recognize the consequences of these choices for how to do PBD effectively.

We have identified in Table 2 a number of different persona kinds from the literature we have reviewed. We do not claim that this list is exhaustive, but we believe it covers most of the kinds of personas present in the current literature. In this section, we provide a brief overview of the persona kinds. Later, we return to these persona kinds and examine how attributes fit with each. Most of the persona kinds described here are associated primarily with one research group. This is not because others do not use these persona kinds (we have made use of Cooperian personas for example); rather, it is because there are usually only a few exemplars in the literature, and they mostly derive from authors who work together.

The two Cooperian personas are derived from publications by Cooper and his associates (i.e. Cooper, 1999; Cronin, 2005). Cooper advocates creating a large number of initial (CI) personas based on in-depth ethnographic research, in an attempt to capture an intuitive understanding of user characteristics. Then, through analysis, the initial personas are condensed together so that there is at most one primary persona for each user kind that is discovered through the analytic process. Thus, the large number of initial personas is transformed into a new, much smaller set of refined, final (CF) personas which are maintained throughout the rest of the design process, but typically discarded once the design activities for that project are complete.

The Pruitt-Grudin (PG) personas (Pruitt \& Adlin 2006; Grudin \& Pruitt, 2002; Pruitt \& Grudin, 2003) are inspired by Cooperian personas, but are a bit different. PG personas are massively data-driven, derived from many different qualitative and quantitative sources, including user studies, surveys, demographic information, etc. Also, there is no culling process, which means that there are often dozens of personas (Chapman \& Milham, 2006 mention over 50 personas used). Furthermore, personas are retained indefinitely, and shared between design projects.

Sinha (2003) personas are also data driven, but the data tend to be primarily quantitative in nature, and are not comprehensive in either the Cooperian or the PG sense. Sinha personas are defined by their creation via quantitative analysis in order to find natural groupings in the data (in Sinha's case via principle component analysis of survey results). Then, personas are written for each of the natural groupings. The problem with this method as it has currently been practiced in the literature is that it is unclear whether the data that can be quantified are really sufficient to represent the full range of relevant user characteristics which are to be compiled into personas.

Norman (2006) describes a form of persona, called ad hoc personas, which is not based on any strong empirical foundation but rather is derived from intuition and experience. Norman does not believe that the intricate details and minutiae of persona descriptions contribute to the core purpose of personas in the design process, which is to foster an "empathetic focus" among the designers (2006). Ad hoc personas need not be real people, only realistic and believable. Norman does not explicitly state that ad hoc personas need to be fictional; their definition is not incompatible with empirically derived personas. Likewise, their use in the design process adheres to that described by Cooper, deriving many of the same benefits.

Djajadiningrat et al. (2000) have developed a variant on the ad hoc persona called extreme characters. Here, the goal is to explore the edges of the design space by exploring behavior which otherwise would not be considered because it violates social norms or expectations. There is still an effort to maintain some level of believability, but the examples are clearly more stereotype-based than experience- based. Because the purpose is to challenge designers' assumptions about the range of human behavior, this is not so much of a problem and, for the purposes of the ontology, we consider them to be simply another form of ad hoc personas: both extreme characters and Norman's accounts of ad hoc personas reflect the intuitions of the designers about users, not actual user groups, thus the limitations on how they can be properly used are the same.

User archetypes (e.g. Dantin, 2005) are very similar to personas but usually are more generic than a persona. A persona is an archetypal user, a hypothetical, representative member of the set of users, whereas a user archetype is a description which defines a set of users. The user archetype is not as precise as a persona; usually being identified by their role or position, rather than a name (e.g. "The Superuser" and "Help Desk" [Egli, 2006], or "Travelling Business Man" [Rönkkö et al., 2004]). However, simply giving a name to a user archetype does not make it a persona. User archetypes are elastic, able to describe several people 


\section{Persona kinds}

Cooperian Initial Personas (CI)

Cooperian Final Personas (CF)

Pruitt-Grudin Personas (PG)

Sinha Personas (S)

Ad hoc Personas (AH)

User Archetypes as Personas (UA)

Marketing Personas (M)

\section{Table 2.}

Persona kinds

simultaneously. The Travelling Business Man in Rönkkö et al. (2004), while being a constant presence throughout the design, changed and evolved over the course of the design, leading to multiple understandings of the target user - precisely one of the things personas were designed not to do.

Marketing personas are not a major focus of this paper. We mention them briefly in order to clarify that personas created for marketing reasons are typically not composed in such a way that they are directly useful for supporting design (Barlow-Busch, 2006). They might be considered data which can be used to inform the creation of other kinds of personas (e.g. PG personas) but, typically, they should not be used in design unless in this complementary manner. Also, their appearance as a single persona kind is probably misleading: we expect that there may be multiple kinds of marketing personas, but we have not analyzed the literature to determine whether this is indeed the case.

\section{PERSONA ATTRIBUTES}

Personas differ by three major variables: (a) the source of the information in the persona (mentioned above), (b) the amount of detail in the persona documentation, and (c) the purpose for which the persona has been constructed. These variables form the source of the persona attributes we identify. The persona attributes and the variables from which they are derived are captured in Table 3. We then apply them to the persona kinds in Table 4. Due to space constraints, we will not review these tables in depth. However, we will comment on some of their contents.

The first variable is the source of the information in the persona. We have created two attributes from this variable: empirical source of data, and the fictional components of the persona. The value of the former is a description of the kind of empirical data that are used in constructing the persona, how they are gathered, etc. The value of the latter is what components can be fictionalized, what must be empirical, and any constraints on the fictions which are created. As should be obvious, the values for the attributes are not quantifiable. Rather, they are descriptions of certain features of the persona kinds.

The empirical source of data attribute specifies what empirical evidence has been used to compose the persona. Thus Cooperian personas are composed primarily from ethnographic data, the PG personas are compiled from a wide range of user studies, and the Sinha personas are compiled from the results of narrow quantitative studies. Ad hoc personas are composed from any of a variety of sources, most of which are of questionable rigor (e.g. intuition), which means the personas are typically more suited for facilitating brainstorming, conversation, or trouble-shooting as opposed to grounding major design decisions.

The fictional components attribute specifies what aspects of the personas are fictional, and, if applicable, why they are justified. Thus, the Cooperian and the PG personas use fictional components sparingly, and only to spark carefully selected intuitions, while the ad hoc personas use fictional components more generously. The User Archetype (UA) personas, on the other hand, may incorporate stereotypes of a user group that are not verified through user research, thus indicating a potential pitfall of that kind of persona.

The amount of detail in the persona description is the second major variable, and it leads to only one attribute: level of detail. Surprisingly, this variable does not factor into discussions in the PBD literature, despite the fact that the amount of detail used to describe personas created by different methods can vary greatly. For example, while the length of the "Foundation Document" that forms the basis of scenario and other communication device construction for PG personas is unspecified, the amount of information it contains suggests that it is quite long, especially if one then includes subsidiary material that is used to specify various instances of the persona (Grudin \& Pruitt, 2002). However, other sources on personas suggest that persona descriptions should be no longer than a single page (e.g. Henry, 2003). Thus, it is clear that the lengths of these descriptions can vary greatly, which in turn determines the amount of detail the persona descriptions can contain.

The third major variable is the use to which the persona is put. With issues of intended use, actual use, consequences of structure for use, etc., this variable is complicated, and leads to a number of different attributes.

The purpose for creating persona attribute describes the intentionality behind constructing the personas. The values in the ad hoc persona cell are defined as follows (see Jones et al., 2008):

1. Propositional: a persona created to exhibit a theoretical set of user characteristics (whether realistic or not); used to interrogate the design.

2. Illustrative: one or more personas created to illustrate different envisioned uses of the design (when persona $A$ uses our design, this is the outcome, when persona $B$ uses our design, that is the outcome). 
3. Intuitive-archetype: create a persona to capture intuitions about a user group and its needs and characteristics before real needs and characteristics have been investigated (e.g. onthe-fly persona creation during a conversation).

The user characteristics attribute represents the space of user characteristics which the persona kind covers. Thus, the CF persona is comprehensive over all relevant dimensions of user characteristics, the PG persona is comprehensive over all the different kinds of user research that has been completed, and the ad hoc persona only covers a narrow focus that is relevant to the activity, problem, or conversation at hand.

The use of persona attribute describes how and why the persona is used and applied. The method of creation attribute describes how the persona is constructed. The values for both of these attributes should be relatively clear. The permanence attribute describes for how long particular personas are retained, with some personas being relatively disposable (e.g. ad hoc and CI personas), others being retained only for a particular design activity (e.g. CF personas), and others being relatively permanent (e.g. PG personas).

\section{PERSONA CHARACTERISTICS}

Finally, we have identified a number of characteristics which particular instances of personas might have (see Table 5). It is important to note that these characteristics apply to particular personas which are instances of any of the kinds mentioned above. Additionally, the kinds of personas are mutually exclusive, while the characteristics of personas are not mutually exclusive, neither with each other, nor with the persona kinds.

One characteristic a persona might have is that it is optimized for teaching. Teaching-optimized personas are created for instructional purposes. Thus they mimic the form of a "real" persona kind, but may be partially or completely fictional in nature. They are created to demonstrate the process of creating a persona and the final form of a persona, but are not necessarily used in any real design project. Teaching personas come from a variety of sources, including real personas sampled from real designs, modified versions of such personas, and ad hoc personas created "on-the-fly" for illustrative purposes.

A persona may also be optimized for communication. Communication-optimized personas are personas whose primary function is to facilitate communication between different stakeholders on the design team. In some respects, every good persona must be communicationoptimized in order to be successful. If personas do not serve as successful boundary objects (Star \& Griesemer, $1989)$ then their worth is debatable. The nature of how the personas are optimized for communication, however, depends on the context of use. Teaching-optimized personas will be optimized for communication between teachers and students; Cooperian final personas will be optimized for communication between those designers who were engaged in ethnographic research in user communities and designers who were not; Pruitt \& Grudin personas will be optimized for communication between designers who are engaged in designing different aspects of a product which will eventually need to be integrated; and many ad hoc personas are created only for facilitating communication. Thus, communication-optimization is a necessary characteristic of a successful persona, thought in most cases not a sufficient one (e.g. ad hoc personas).

Rönkkö et al. (2004) provide a fascinating study of the failure of personas in use in a particular commercial context. However, we believe that despite demonstrating the limitations of the persona method, it also shows its power. In the study the use of personas highlighted

\section{Persona attributes}

\begin{tabular}{|c|c|c|}
\hline Variable & Attribute & Description \\
\hline \multirow[t]{2}{*}{ Source of data } & Empirical source of data & $\begin{array}{l}\text { The kind of empirical data that are used, how they are gathered, what } \\
\text { aspects of the persona must be supported by empirical data, etc. }\end{array}$ \\
\hline & Fictional components & $\begin{array}{l}\text { What aspects of the personas are fictional, why they are justified, con- } \\
\text { straints, etc. }\end{array}$ \\
\hline Detail of description & Detail of description & $\begin{array}{l}\text { The amount of detail provided in the persona description, both what is } \\
\text { necessary, and what is too much }\end{array}$ \\
\hline \multirow[t]{5}{*}{ Intended purpose } & Purpose for creating persona & The intent behind creating persona what it is supposed to accomplish \\
\hline & Use of persona & How the persona is used and applied \\
\hline & Method of creation & How the persona is created \\
\hline & Permanence & How permanent the persona is supposed to be \\
\hline & User characteristics & $\begin{array}{l}\text { The space of user characteristics which the persona kind covers, how } \\
\text { comprehensive is it, what kinds of characteristics can it cover, etc. }\end{array}$ \\
\hline
\end{tabular}

Table 3.

Persona kind attributes 


\begin{tabular}{|c|c|c|c|c|c|c|c|c|}
\hline Empirical source & Ethnographic observation & $x$ & $x$ & $\mathrm{x}$ & & & & \\
\hline \multirow[t]{8}{*}{ of data } & Anecdotes & $x$ & $x$ & $x$ & & $x$ & $\mathrm{x}$ & \\
\hline & Other personas & & $\mathrm{x}$ & $\mathrm{x}$ & & & & \\
\hline & Qualitative data & & & $\mathrm{x}$ & & & & \\
\hline & Quantitative data & & & $\mathrm{x}$ & $\mathrm{x}$ & & & $\mathrm{x}$ \\
\hline & User surveys & & & $\mathrm{x}$ & $\mathrm{x}$ & & & $\mathrm{x}$ \\
\hline & Market research & & & $\mathrm{x}$ & $\mathrm{x}$ & & & $\mathrm{x}$ \\
\hline & Designer intuition & & & & & $\mathrm{x}$ & $\mathrm{x}$ & \\
\hline & Personal experience & & & & & $\mathrm{x}$ & $x$ & \\
\hline \multirow[t]{4}{*}{ Fictional components } & Never used & & & & $x$ & & & $\mathrm{x}$ \\
\hline & Only when capture user needs viscerally & $\mathrm{x}$ & $\mathrm{x}$ & $\mathrm{x}$ & & & & \\
\hline & Stereotypes of user groups & & & & & $x$ & $\mathrm{x}$ & \\
\hline & Readily used to communicate ideas & & & & & $\mathrm{x}$ & & \\
\hline \multirow[t]{4}{*}{ Level of detail } & Long description, highly detailed & & & $x$ & & & & \\
\hline & Enough detail to spark designer intuitions, no more & $x$ & $x$ & & & $\mathrm{x}$ & & \\
\hline & Short, concise & & & & $\mathrm{x}$ & & & \\
\hline & Varies & & & & $\mathrm{x}$ & & $\mathrm{x}$ & $\mathrm{x}$ \\
\hline \multirow[t]{10}{*}{ Purpose and use } & Leverage designer intuitions & $\mathrm{x}$ & $\mathrm{x}$ & $\mathrm{x}$ & $x$ & $x$ & $x$ & \\
\hline & Put a human face on quantitative data & & & $\mathrm{x}$ & $\mathrm{x}$ & & & $\mathrm{x}$ \\
\hline & Consider possibly hypothetical use cases & & & & & $x$ & & \\
\hline & To generate CF personas & $\mathrm{x}$ & & & & & & \\
\hline & Prevent designers from designing for themselves & $\mathrm{x}$ & $x$ & $x$ & $x$ & & & \\
\hline & Facilitate communication & $x$ & $x$ & $x$ & $x$ & $x$ & & \\
\hline & Make intuitions explicit & $\mathrm{x}$ & & & & $x$ & & \\
\hline & Describe major user groups & & & & & & $x$ & $\mathrm{x}$ \\
\hline & Better understand the market & & & & & & & $\mathrm{x}$ \\
\hline & Illustrate consequences & & & & & $\mathrm{x}$ & & \\
\hline \multirow[t]{7}{*}{ Method of creation } & $\begin{array}{l}\text { Inscribe experiences of ethnographic immersion } \\
\text { and observation }\end{array}$ & $\mathrm{x}$ & & & & & & \\
\hline & Distill important user attributes & & $\mathrm{x}$ & $x$ & & & & $\mathrm{x}$ \\
\hline & Exhaustive composite of user-study results & & & $\mathrm{x}$ & & & & \\
\hline & Quantitative identification of similarities & & & & $x$ & & & $\mathrm{x}$ \\
\hline & Draw upon intuitions and past experiences & & & & & $x$ & & \\
\hline & Evolves over time, enriched and changed & & & & & & $x$ & \\
\hline & Created on the fly & & & & & $\mathrm{x}$ & & \\
\hline \multirow[t]{4}{*}{ Permanence } & Disposable & $\mathrm{x}$ & & & & $\mathrm{x}$ & & \\
\hline & Retained through life of design & & $x$ & & & $\mathrm{x}$ & & \\
\hline & Changes and evolves over life of design & & & & & & $\mathrm{x}$ & \\
\hline & Retained as long as accurate and useful & & & $\mathrm{x}$ & $\mathrm{x}$ & & & $\mathrm{x}$ \\
\hline \multirow[t]{6}{*}{ User characteristics } & All relevant details of a user group; comprehensive & & $\mathrm{x}$ & $\mathrm{x}$ & & & & \\
\hline & Particular, salient user characteristics & $\mathrm{x}$ & $\mathrm{x}$ & & & $\mathrm{x}$ & & \\
\hline & User characteristics revealed by survey (artificial) & & & $\mathrm{x}$ & $\mathrm{x}$ & & & $\mathrm{x}$ \\
\hline & User demographic information & & & $\mathrm{x}$ & & & & $\mathrm{x}$ \\
\hline & Stereotypical user characteristics (non-empirical) & & & & & $x$ & $x$ & \\
\hline & User characteristics of a particular, expected kind & $\mathrm{x}$ & $\mathrm{x}$ & $\mathrm{x}$ & $x$ & $x$ & $\mathrm{x}$ & $\mathrm{x}$ \\
\hline
\end{tabular}

Table 4.

Persona attribute values 
some irreconcilable differences between various design stakeholders. Using personas clearly did not reconcile these differences, but it does seem that it made them abundantly clear. In this case personas enforced perhaps excessive clarity in communication across disciplinary boundaries. The fact that marketing people want and need to talk only about successes - the desirability of the product to be built to persuade people to purchase it and integrate it into their lives - runs counter to the need of the UI designer to focus almost exclusively on failures to try and fix. This is very often the case, and is not just an artifact of the particular organization studied. Typically marketing and UI speak different languages and so can work together despite these contradictions because they are not made so crystal clear as to be problematic. Persona use was so powerful in communication that the different needs and uses were made uncomfortably visible for all to see.

A persona may have the characteristic of being a "twin" of another persona being used in the same design context. A persona twin-set is a collection of two or more personas which superficially appear to be very different, but are very similar or identical with respect to particular aspects of the design problem. Especially from a Cooperian perspective, using multiple personas is supposed to expand the designer's understanding through investigating a plurality of user characteristics and uncovering as many issues as possible (Cooper, 1999; Cronin, 2005). Twin personas, however, limit what the designer can learn by providing redundant information.

Figure 1 contains excerpts from two personas created by a group of students on one of our courses. The students were designing a web-based customer-relationsmanagement system. John and Paul appear to be very different, and indeed can be useful in generating and evaluating design ideas. However, what makes them twin personas is their essential similarity when it comes to the price and essential functionality of the software. We are not arguing that John and Paul are not useful personas, but personas are supposed to represent as diverse a set of characteristics and needs as possible to maximize the coverage of the design space.

Interestingly, twin personas can be used to identify the designers' assumptions (both implicit and explicit) and design priorities. The aspects with regard to which several personas are similar or identical can be useful pointers in identifying the unquestioned assumptions of the designer. In the above example, John and Paul illustrate the designers' prioritization of price and simplicity in design.

Some personas have the characteristic of being politically correct (PC) in that they break stereotyped associations. Such a persona is stereotypically discordant with other aspects of the persona, i.e. age with job seniority, gender with job type, habits with socioeconomic status, etc. Cooper $(1999$, p. 128) has stated that, "All things being equal, I will use people

\section{Persona kinds}

Teaching-optimized

Communication-optimized

Twin

PC

Elastic

Promotional

Unitary-interpretation

Table 5.

Persona instance characteristics

of different races, genders, nationalities, and colors as personas. However, I try not to play against type because this can confuse everyone. Stereotypical personas are more effective if the stereotype lends more credence to the persona. My goal here is not to be politically correct but to get everyone to believe that my personas are real." These comments have rubbed some designers up the wrong way, causing them to overemphasize political correctness at the expense of believability. In her criticism of personas as a design method, Marshall (2003) uses an anecdote of a colleague's young, attractive, female truck driver persona. The persona was not believable to Marshall (or us), thus detracting from its value as a design tool. While creating a persona with PC characteristics can be useful for making a persona more memorable, it is important not to construct the character of the persona such that it no longer represents actual needs and characteristics of the users it is designed to represent.

As the world changes, stereotypes change (e.g. gender distribution among nurses, teachers, truck drivers, etc.). However, for a persona to be useful, it needs to reflect the world as it is, not the world as one might like it to be. The power of a persona resides in its memorability and ease of consideration by designers. If stereotypedefying personas get in the way of the reasoning process, then, despite their desirability in reminding us of the need to promote diversity, they will fail to support effective user-centered design. For example, one might legitimately want to challenge the stereotype of older people being reluctant to embrace new technologies. It is certainly the case that many older people are eager early adopters of computer technology, but an empowered persona of a computer savvy senior who not only effortlessly learns new technologies but actively participates in open source software development may not be very useful in the design process. Personas who have problems with current technologies and can even be envisaged struggling with the application being designed are often more helpful in design than personas who heroically and insightfully adopt and adapt technologies.

If one can foresee gender distributions (for example) of a particular user type changing over time, it might be useful to create stereotype- breaking personas to explore how the design space might change. $0 r$, it might be useful to test the edges of the design space with 


\section{John}

John's business deals with computer networking. His company has five employees. John and his employees are always on the go. They are traveling all the time trying to gain and manage their clients. While the company is getting better, they are in need of a software package that allows them to keep track of their contacts. Microsoft Dynamics is a good solution to this problem but it cost too much for the company.This growing company is in need of a program that can manage its contacts but also be at a reasonable price.

\section{Paul}

Paul sells items online through Ebay. With a variety of items, Paul has racked in over US\$30,000. Currently after selling items to a buyer, it is hard to stay in contact with that particular client. If Paul has five different former clients who like a particular type of product, it becomes a hassle in trying to contact these people to try and sell them these items. Paul could buy A.C.T to help him manage his clients but A.C.T is very expensive and would severely cut into his revenues.

Figure 1.

Twin personas: John and Paul.

extreme characters (Djajadiningrat et al., 2000). But such persona construction exercises should be based on a conscious evaluation of relevant considerations, not a designer's whim.

Some personas have the characteristic of being elastic, in that they could be about nearly anybody. Like Cooper's (1999) elastic user, the elastic persona can be stretched until it is one step shy of being the "any person". The power of the precision of personas is that it puts sharp constraints on the possible behaviors of the hypothetical user. Thus, designers can avoid the trap of morphing their understanding of the user into any desired form that is convenient.

Figure 2 contains persona Sophie 32 from Goossens (2006), a persona we consider to be elastic in nature. We have provided two doppelgangers for Sophie immediately below the original description (see Figure 2). Sophie Jenkins is a more concrete interpretation of who Sophie might be. With the change of a single pronoun (i.e. "his") in the original persona description, Sophie Cook is as plausible an interpretation of Sophie 32 as Sophie Jenkins.

The key thing to remember in the problem with the elastic user is that a designer is free to say both "I'm a user, and I would do this", and "I wouldn't do this, but I know some user would" as justification for the design decisions he/she makes. Personas are meant to prevent such elastic conceptions of users, by providing a particular, fictional user whose preferences and attitudes are clear. If a persona is too general (too elastic), then it will fail in this regard. However, elastic personas may be useful for practicing Dix et al.'s (2006) method of bad design. By trying to create an elastically infallible persona and having it use some excessively complex and inappropriate system, the very preposterousness of the situation can expose previously uncovered aspects of the design space.

This criticism indicates that a positive characteristic of non-elastic personas is having a unitary interpretation when applied to a design. Personas with unitary interpretations are specific enough that the preferences and attitudes of the persona are so clear that designers will intuitively and unambiguously understand what the persona's preferences are, for any kind of design decision which the persona is geared to address. This does not mean that the persona may not "care" about a particular design feature (e.g. a persona might be agnostic about interface color-schemes), but only that for the design decisions which are important the persona will have clear preferences which the designers can intuit.

The unitary-interpretation characteristic of a persona is probably more of an ideal to aim for, rather than something for which one can specifically design for. However, it suggests that a persona that is found to have multiple interpretations ought to be revised based on the empirical evidence which was used to create the persona. Thus, lack of unitaryinterpretation can be treated as a warning sign that a persona is poorly constructed.

Personas can also have the characteristic of being promotional. Promotional personas are not constructed out of any grounded understanding of users or usecontexts, but rather based on a designer's preconceived notions of what the design ought to be. Promotional personas are the Trojan Horses of PBD. They allow a designer to sneak in his or her personal preferences using the form "something based on user-research". A designer wants the design to support a particular feature or to evolve in a particular way, and rather than researching that idea and being open to alternatives, he or she constructs a persona which is later used to justify design decisions and advance the designer's objectives. This gives the designer the false sense of rigor or diligence and of having done user-centered design, when in reality all he/she has done is create a design for him/ herself and his/her (often false) understanding of what users want or need and of what they do.

One example of a promotional persona comes from Cronin (2005); he describes Penelope, a persona created by a client on a project. Penelope was someone who "spent her day looking around for novel ways to use enterprise software". Cronin quickly adds that Penelope was "not based on user research and did not represent the needs of any users we [the Cooper design consultants] encountered" (Cronin, 2005, 9). The client who created Penelope had an idea of what the software would allow him to do, and he had already started formulating ideas of how he wanted to use it. The Penelope construct was his way of trying to bias the design towards his vision, by seeding his ideas into the design discussion. 


\begin{tabular}{|c|}
\hline Sophie (32) \\
\hline $\begin{array}{l}\text { Sophie lives in one of the big cities and owns a first or second } \\
\text { house/apartment together with her partner. She surfs the Web } \\
\text { on a broadband connection and both the computer and the } \\
\text { Internet play a central role in her daily life. She plays a couple } \\
\text { of games on the Internet every week if her busy schedule } \\
\text { permits it. }\end{array}$ \\
\hline $\begin{array}{l}\text { Sophie lives a busy life, combining her work and socializing } \\
\text { with her many friends and close relatives. Her partner is also } \\
\text { working his way up the ranks and they both have an } \\
\text { above-average education. Sophie is ambitious but also longs to } \\
\text { start her own family. Playing games gives her a moment of rest } \\
\text { in a busy day. In their spare time Sophie and her partner like to } \\
\text { go out, preferably with friends or family. }\end{array}$ \\
\hline
\end{tabular}

Sophie Jenkins

Sophie lives with her husband Tom in a beautiful Victorian in San Francisco's Mission Hill district. Sophie and Tom recently married, and Sophie is still adjusting to her partnered life. She loves playing World of Warcraft, and has lots of friends in game, although her new job as Project Manager at Wijjetz Software has severely cut into her gaming time. Tom doesn't understand Sophie's obsession with Orcs and Druids, and teases her for playing "childish games". Tom's teasing makes Sophie feel guilty about playing WoW for too long, so she reeps her gaming to about five hours a week, usully invested in long sessions on Sunday mornings while Tom is sleeping in.

Sophie and Tom have lots of "couple friends", and usually spend Friday nights having cocktails and dinner with at least one other couple. Sophie's sister Laura still lives at home with her parents in the East Bay and Sophie tries to get out to visit her family at least once a month. Sophie's brother Dan moved to Chicago a couple of years ago, and while they haven't seen each other in a long time, they do meet up in World of Warcraft almost every week.

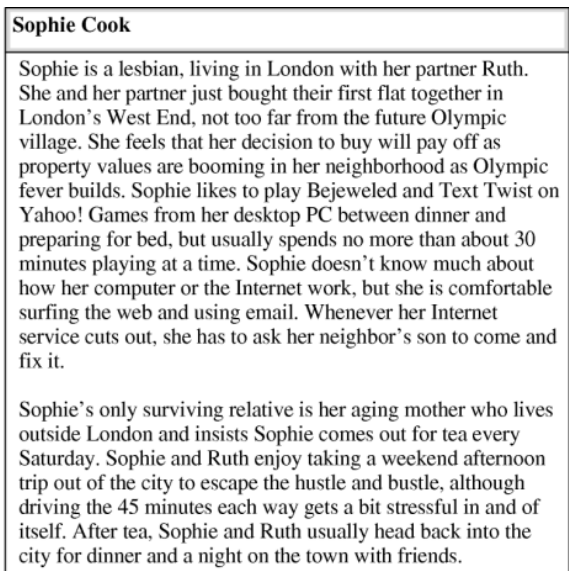

Figure 2

Sophie and her doppelgangers.

\section{CONCLUSIONS}

Criticisms of persona-based design need to be understood in the light of the kind of persona and the kind of use that they are critiquing. To do that it is important to understand the diversity of methods, approaches, and uses that are all described by the same term "persona". By doing so we can try and understand whether problems identified apply to the context of use of personas that was studied, the way the method was used, appropriately or not, and critically which method was used. In this way we can help advance refinements, extensions, and improvements in the method, and also help understand what is causing personas to work effectively in one use-context, and less so in another.

\section{ACKNOWLEDGEMENTS}

The authors would like to thank the many students they have had in their classes who have struggled to understand personas with them. Their insights have been valuable, and the authors' informal observations of their actions have led them to a much richer understanding of personas, both in how they work well, and in how they can be inadvertently misused. They would also like to thank the many reviewers whose comments on various versions of this paper have been invaluable for helping them construct this final draft. Finally, they would like to thank the Research Writing Group at the Graduate School of Library and Information Science at the University of Illinois at Urbana Champaign. Their feedback was vital for helping the authors reconceptualize their project as more than a critique of critiques, or the generation of a simple typology.

\section{NOTE}

1. We use the term "ontology" loosely here. We do not mean to imply that what we have developed should be considered as a formal, precise account. Rather we are trying to avoid some words such as taxonomy or typology that have more specific implications which we do not intend our work to carry.

\section{REFERENCES}

Aquino, P. T., \& Filgueiras, L. V. L. (2005). User modeling with personas. Proceedings of the 2005 Latin American conference on Human computer interaction (Latin AmericanCHI 2005). Cuernavaca, Mexico: ACM Press.

Barlow-Busch, R. (2006). Marketing versus design personas. In J. Pruitt, \& T. Adlin (Eds.), The Persona Lifecycle: Keeping people in Mind During Product Design (pp. 602-641). Amsterdam, Netherlands: Morgan Kaufmann Press; Elsevier.

Blomquist, A., \& Arvola, M. (2002). Short papers: Personas in action: ethnography in an interaction design team. Proceedings of the second Nordic conference in Humancomputer interaction (NordiCHI 2002). Aarhus, Denmark: ACM Press.

Carroll, J. M. (2000). Making use: scenario-based design of humancomputer interactions. Cambridge, MA: MIT Press.

Chapman, C. N., \& Milham, R. P. (2006). The personas' new clothes: methodological and practical arguments against a popular method. In Proceedings of the Human Factors and Ergonomics Society 50th Annual Meeting (pp. 634-636). San Francisco, CA: Human Factors and Ergonomics Society.

Cooper, A. (1999). The inmates are running the asylum. Houndmills, UK: Macmillan.

Cooper, A. (2003). The origin of personas. Cooper Journal of Design. Retrieved 27 August 2008 from http://www.cooper. com/journal/2003/08/the_origin_of_personas.html

Cronin, D. (2005). Early and often: how to avoid the design revision death spiral. In Proceedings of the 2005 conference on Designing for User Experience. San Francisco, CA: ACM Press.

Dantin, U. (2005) Application of personas in user interface design for educational software. In Proceedings of the 7th Australasian conference on Computing education. Newcastle, NSW: ACM Press.

Dix, A. Ormerod, T., Twidale, M.B., Sas, C., Gomes da Silva, P.A., \& McKnight, L. (2006). Why bad ideas are a good idea. In Proceedings of the First Joint BCS/IFIP WG13.1/ ICS/EUCONVIVIO HCI Educators' Workshop "HCIEd.2006-1 inventivity: Teaching theory, design and innovation in $\mathrm{HCl}$ ". Ballina/Killaloe, Ireland: Interaction Design Centre. 
Djajadiningrat, J. P., Gaver, W. W., \& Frens, J. W., (2000). Interaction relabelling and extreme characters: Methods for exploring aesthetic interactions. DIS ‘00, Brooklyn, New York: ACM Press.

Egli, C. (2006). Example personas from real projects: mentor graphics. In J. Pruitt, \& T. Adlin (Eds.), The persona lifecycle: Keeping people in mind during product design (pp. 682-690). Amsterdam, Netherlands: Morgan Kaufmann Press; Elsevier.

Goossens, E. (2006). Example personas from real projects: Zylom Media Group. In J. Pruitt, \& T. Adlin (Eds.), The persona lifecycle: Keeping people in mind during product design (pp. 682-690). Amsterdam, Netherlands: Morgan Kaufmann Press; Elsevier.

Grudin, J. (2006). Why personas work: The psychological evidence. In J. Pruitt, \& T. Adlin (Eds.), The persona lifecycle: Keeping people in mind during product design (pp. 642-664). Amsterdam, Netherlands: Morgan Kaufmann Press; Elsevier.

Grudin, J., \& Pruitt, J. (2002). Personas, participatory design and product development: An infrastructure for engagement. PDC 2002. Malmo, Sweden: Computer Professionals for Social Responsibility.

Henry, S. L. (2003). Just Ask: Integrating Accessibility Throughout Design. UIAccess.com. Retrieved 27 August 2008 from http://www.uiaccess.com/accessucd/personas. html

Jones, M. C., Floyd, I. R., \& Twidale, M. B. (2008). Teaching design with personas. In Proceedings of the Human Computer Interaction in Education 2008 (HCIEd 2008), Conference, Rome, Italy: Tor Vergata University.

Lafreniere, D. (1996). CUTA: A simple, practical low-cost approach to task analysis. Interactions, 3(5), 35-39.

Lowgren, J., \& Nagai, Y. (2008). Call for Contributions for a Special Issue of the journal Artifact: Issues in Design Research and Design Research Methods. Retrieved 27 August 2008 from http://www.tandf.co.uk/journals/cfp/ saifcfp1.pdf

Marshall, C. (2003). The trouble with scenarios and personas. Retrieved 27 August 2008 from http://www.csdl.tamu.edu/ marshall/mc-scenarios-personas.html

Norman, D. (2006). Ad hoc personas \& empathetic focus. In J. Pruitt, \& T. Adlin (Eds.), The persona lifecycle: Keeping people in mind during product design (pp. 154-157). Amsterdam, Netherlands: Morgan Kaufmann Press; Elsevier.

Portigal, S. (2008). Persona non grata. Interactions, 15(1), 72-73.

Pruitt, J., \& Adlin, T. (2006). The persona lifecycle: Keeping people in mind during product design. Amsterdam, Netherlands: Morgan Kaufmann Press; Elsevier.

Pruitt, J., \& Grudin, J. (2003). Personas: Practice and theory. DUX 2003. San Francisco, CA: ACM Press.

Quesenbery,W. (2006). Storytelling and narrative. In J. Pruitt, \& T. Adlin (Eds.), The persona lifecycle: Keeping people in mind during product design (pp. 520-555). Amsterdam, Netherlands: Morgan Kaufmann Press; Elsevier.
Rönkkö, K. (2005). An empirical study demonstrating how different design constraints, project organization and contexts limited the utility of personas. Proceedings of the 38th Hawaii International Conference on System Sciences. Waikoloa Village, Hawaii: IEEE.

Rönkkö, K., Hellman, M., Kilander, B., \& Dittrich, Y. (2004). Personas is not applicable: Local remedies interpreted in a wider context. PDC 2004. Torento: Computer Professionals for Social Responsibility.

Rosson, M. B., \& Carroll, J. M. (2002). Usability engineering: Scenario-based development of human-computer interaction. San Francisco, CAA: Morgan Kaufmann Publishers.

Sheard, S.A. (2003). Life Cycle of a Silver Bullet. CrossTalk, The Journal of Defense Software Engineering. July 2003. Retrieved from: http://www.stsc.hill.af.mil/ Crosstalk/2003/07/Sheard.html

Sinha, R. (2003). Personas development for information-rich domains. CHI 2003. Fort Lauderdale, FL: ACM Press.

Star, Susan Leigh, \& Griesemer, James (1989). Institutional ecology, "translations", and coherence: Amateurs and professionals in Berkeley's Museum of Vertebrate Zoology, 1907-1939. Social Studies of Science, 19, 387-420.

Williams, K. L. (2006). Personas in the design process: a tool for understanding others. Master's thesis, Georgia Institute of Technology.

Published online 28 November 2008

ISSN 1749-3463 print/ ISSN 1749-3471

DOI: $10.1080 / 17493460802276836$

(C) 2008 Artifact 